\title{
IMPLEMENTAÇÃO DE POLÍTICAS PÚBLICAS PARA O TRATAMENTO DOS PROBLEMAS DE SAÚDE MENTAL DECORRENTES DA PANDEMIA DO COVID-19
}

\author{
Anna Carolina Amorim da Costa ${ }^{1}$
}

RESUMO: Segundo a Organização Mundial de Saúde (OMS), a ansiedade afeta I8,6 milhões de brasileiros e os transtornos mentais são responsáveis por mais de um terço do número de pessoas incapacitadas nas Américas. O presente artigo analisa o aumento das diversas doenças mentais após o início da pandemia Covid-ı́ e, diante das graves conseqüências que a longo prazo impactarão na sociedade, evidencia a necessidade de implementação de políticas públicas municipais adequadas e eficazes para o reestabelecimento da saúde mental dos cidadãos. Tamanha é a importância que deve ser dada ao tema, que a Organização Mundial da Saúde (OMS) inseriu a "síndrome do esgotamento profissional", chamada como Burnout, no rol de doenças ocupacionais desde I e janeiro de 2022, doença que pode ensejar no afastamento do trabalho. É crucial o tratamento dessas doenças para o bem-estar da coletividade e para o futuro das companhias públicas e privadas, que terão que redobrar os cuidados com a saúde mental de seus servidores e colaboradores.

Palavras-chave: Covid-ı. Transtornos mentais. Políticas públicas de saúde.

ABSTRACT: According to the World Health Organization (WHO), anxiety affects 18.6 million Brazilians and mental disorders are responsible for more than a third of the number of disabled people in the Americas. This article analyzes the increase in various mental illnesses after the beginning of the Covid-ig pandemic and, given the serious consequences that will impact society in the long term, highlights the need to implement adequate and effective municipal public policies for the reestablishment of mental health. of citizens. Such is the importance that must be given to the topic that the World Health Organization (WHO) has inserted the "professional burnout syndrome", called Burnout, in the list of occupational diseases since January $\mathrm{I}, 2022$, a disease that can give rise to the absence from work. The treatment of these diseases is crucial for the well-being of the community and for the future of public and private companies, which will have to redouble the care with the mental health of their servers and collaborators.

Keywords: Covid-ı. Mental disorders. Public health policies.

\footnotetext{
${ }^{1}$ Pós-graduada em Gestão Pública Municipal pela Universidade Federal do Paraná. Pós-graduada em Direito Público pelo Centro Universitário Leonardo da Vinci, Graduada em Direito pela Universidade Estadual de Ponta Grossa . Advogada e Servidora Pública ocupante do cargo de Procuradora da Câmara Municipal de Palmeira desde 2oro. E-mail: annacarolina_amorim@yahoo.com.br.
} 


\section{INTRODUÇÃO}

A Organização Mundial da Saúde (OMS) tem destacado que transtornos psiquiátricos devem ser considerados uma preocupação mundial durante a pandemia da covid-ı. Segundo essa Organização, a ansiedade afeta ı8,6 milhões de brasileiros e os transtornos mentais são responsáveis por mais de um terço do número de pessoas incapacitadas nas Américas. Apesar de antigas, as doenças mentais estão ganhando novos termos e nova proporção de importância em razão desse período de pandemia que assolou o mundo. A covid-ı́ não foi o maior motivo de afastamento de saúde no ambiente de trabalho no último ano; uma pesquisa ${ }^{2}$ mostrou que em primeiro lugar estão as doenças do sistema osteomuscular e do tecido conjuntivo, em segundo os transtornos mentais, em terceiras lesões e em quarto a referida doença infecciosa. ${ }^{i}$

A Covid-ı́ fez não só com que esses transtornos se agravassem, mas também trouxe novas questões à tona, como o fato de obrigar o mundo a lidar com o isolamento, com o lockdown, com as perdas financeiras e pessoais, com o medo generalizado, com a insegurança e diversos outros 'monstros' que passaram a assombrar o dia a dia das pessoas.

É notório que estamos perante um caso de direitos humanos que atingiu o mundo todo, sendo possível identificar a saúde pública como ideal comum a atingir por todos os povos e todas as nações, a fim de que todos os indivíduos e todos os órgãos da sociedade se esforcem por desenvolver a busca desse direito e das liberdades e por promover, por medidas progressivas de ordem municipal à ordem internacional, o seu reconhecimento e a sua aplicação efetiva. Conforme bem coloca Joaquim Herrera Glores:

Do ponto de vista de uma nova teoria, as coisas não são tão aparentemente simples. Os direitos humanos, mais que direitos propriamente ditos, são processos; ou seja, o resultado sempre provisório das lutas que os seres humanos colocam em prática para ter acesso aos bens necessários para a vida. ${ }^{\text {ii }}$

São diversos os sintomas, os diagnósticos e as consequências; afetam crianças, adultos e idosos; impactam o humor, a disposição, a esperança, os objetivos de vida e a vontade de idealizar e realizar projetos. Portanto, os resultados iminentes do crescimento desses distúrbios são seriamente preocupantes a longo prazo. A recuperação desse quadro

\footnotetext{
${ }^{2}$ Levantamento realizado pela $\mathrm{B}_{2} \mathrm{P}$, consultoria especializada no acompanhamento e gestão de funcionários afastados por razões médicas, da It'sSeg Company.
} 
de saúde pública é um dever da administração, garantia constitucional de direitos humanos.

Quadros de ansiedade e depressão já existentes foram intensificados, quadros novos se proliferaram e a busca por tratamento aumentou de forma considerável, algumas pessoas buscando terapias e outras medicamentos; isso considerando o número efetivo de diagnósticos, prescrição e venda de remédios, pois existe um número ainda maior, visto que que muitas pessoas sequer buscam ajuda ou realizam tratamento.

Entre os elementos que colaboraram para o aumento desses casos está a diminuição do convívio social, o excesso de trabalho para uns e a perda do emprego para outros, a impossibilidade de ter momentos de lazer como antes, o medo, a insegurança, os problemas financeiros, o contágio pelo Coronavírus e o peso do luto por perder algum ente querido sem ao menos a possibilidade de despedida.

Importante ressaltar que muitos trabalhadores da área da saúde foram acometidos da chamada fadiga pandêmica e de outras doenças mentais, sendo imprescindível que o poder público adote as medidas necessárias para o tratamento e a recuperação desses profissionais, já que são eles que cuidam da saúde da população. Fadiga pandêmica é a terminologia recentemente adotada pela OMS para designar o cansaço e o esgotamento físico e mental provocados pela pandemia. ${ }^{i i i}$

Essa retomada da saúde pública em detrimentos dos efeitos da pandemia é uma das tarefas mais importantes e complexas dos direitos humanos, requer planejamento, estudo, tempo e políticas públicas eficazes. Ela é medida crucial e urgente, devendo ser tratada como prioridade.

Com base nessas informações, evidencia-se a relevância do estudo e da pesquisa, a fim de proporcionar suporte ao poder público, na implantação de políticas públicas eficazes na retomada do reequilíbrio da saúde mental da população.

Segundo um estudo publicado em o6 de abril de 2021 na revista Lancet Psychiatry, uma em cada três pessoas que tiveram Covid-ıg recebeu um diagnóstico de doença mental ou neurológica em um período de seis meses após a infecção; o estudo contou com a participação de 236.379 mil pacientes e incluiu pacientes maiores de dez anos de idade que foram infectados com o coronavírus depois de 20 de janeiro de 2020 e estavam vivos em 13 
de dezembro de 2020, a maioria norte-americanos. Antes desse estudo ser publicado, outra pesquisa já havia detectado dados parecidos: em 2020, os mesmos pesquisadores encontraram $20 \%$ dos sobreviventes do novo coronavírus com o desenvolvimento de algum problema psiquiátrico após três meses da alta de internação. iv

Diante da situação pandêmica que o mundo se encontra há aproximadamente dois anos e diante das novidades diárias que surgem e das novas ondas da doença que emergem em diversos países a cada semana, não se pode afirmar que a pandemia está perto de terminar.

De tal forma, é importante analisar esse índice de doenças e distúrbios mentais na população em geral, sobretudo nos próprios trabalhadores que atuam na prestação do serviço de saúde pública municipal, para que seja possível tratá-los, buscando a retomada e o equilíbrio da saúde mental, considerada essencial para o bem estar no qual o indivíduo desenvolve suas habilidades pessoais, consegue lidar com os estresses da vida, trabalhar de forma produtiva e encontrar-se apto a dar sua contribuição para sua comunidade.

\section{IMPACTO DA PANDEMIA NA SAÚDE MENTAL}

Todos os setores e aspectos são impactados quando se tem uma pandemia mundial. Geralmente eles estão todos interligados e um afeta no outro, de forma direta ou indiretamente.

No cenário atual, a pandemia desencadeou também uma crise econômica, a qual, conforme afirma o autor Fried ${ }^{\mathrm{v}}$, é responsável pelo considerável aumento dos índices de suicídio e das taxas de transtornos mentais, além do crescimento de problemas psicossomáticos. Ainda, segundo Reeves, McKee e Stuckler ${ }^{\mathrm{vi}}$ choques econômicos podem piorar a saúde mental e levar ao suicídio, principalmente devido ao desemprego (o suicídio é 2,5 vezes mais comum em desempregados) e ao endividamento.

Os diagnósticos mais comuns encontrados nos sobreviventes da Covid-19 foram: ansiedade ( $17 \%)$, transtornos de humor (I4\%), abuso de substâncias (7\%) e insônia (5\%); doenças neurológicas também foram diagnosticadas: AVC (acidente vascular cerebral) isquêmico $(2,1 \%)$, demência $(0,7 \%)$ e hemorragia cerebral $(0,6 \%)$. Nos pacientes que tiveram um quadro grave de Covid, foram diagnosticadas doenças neurológicas ou 
psiquiátricas em aproximadamente $34 \%$ dos sobreviventes, sendo o percentual de $38 \%$ naqueles que receberam atendimento hospitalar, de $46 \%$ nos que ficaram internados em UTI e 62\% nos que tiveram encefalopatia durante o período da infecção. ${ }^{\text {vii }}$

De acordo com outra pesquisa internacional conduzida pelo UNICEF e pelo Gallup ${ }^{3}$, resultados preliminares com crianças e adultos em 2I países apresentaram uma prévia da Situação Mundial da Infância 2021: em média, um em cada cinco adolescentes e jovens de 15 a 24 anos entrevistados (19\%) disse que, muitas vezes, se sente deprimido ou tem pouco interesse em fazer coisas. Enquanto a covid-19 está perto de chegar a seu terceiro ano, o impacto sobre a saúde mental e o bem-estar de crianças e jovens continua pesando muito. Segundo os últimos dados disponíveis do UNICEF, globalmente, pelo menos uma em cada sete crianças foi diretamente afetada por lockdowns, enquanto mais de 1,6 bilhão de crianças sofreram alguma perda relacionada à educação. A ruptura com as rotinas, a educação, a recreação e a preocupação com a renda familiar e com a saúde estão deixando muitos jovens com medo, irritados e preocupados com seu futuro. (Taquet Maxime, Geddes John R, Husain Masud, Luciano Sierra, Harrison Paul J., 2021)

Ou seja, além dos diagnósticos efetuados em pessoas que tiveram Covid, devemos considerar também as pessoas que não foram infectadas pelo vírus, mas que, em decorrência da pandemia, desencadearam alguma dessas doenças, tendo em vista os diversos fatores que influenciaram a vida dos cidadãos: isolamento social, luto, perda do potencial financeiro, medo, desemprego, afastamento dos amigos, diminuição do tempo e do espaço de lazer, entre outros.

Dados da Federação Brasileira das Redes Associativistas e Independentes de Farmácias (Febrafar) mostram que as vendas de antidepressivos e benzodiazepínicos (os populares calmantes vendidos com receita) aumentaram em média $24 \%$ em 2020.

Tamanha é a importância do tema, que a Organização Mundial da Saúde (OMS) inseriu a "síndrome do esgotamento profissional", chamada como Burnout, no rol de doenças ocupacionais desde I $\mathrm{o}$ de janeiro de 2022, doença que recebeu o CID II e poderá ensejar no afastamento do trabalho. Como consequência, as empresas - tanto públicas quanto

\footnotetext{
${ }^{3}$ Empresa de pesquisa de opinião dos Estados Unidos, fundada em 1930 pelo estatístico George Gallup.
} 
privadas - terão que redobrar os cuidados com a saúde mental de seus servidores e colaboradores.

Ainda de acordo com estudos realizados, a concessão de auxílio-doença e aposentadoria por invalidez por transtornos mentais e comportamentais bateu recorde em 2020, o número de casos de Burnout disparou após a pandemia e, segundo uma pesquisa da $\mathrm{B}_{2} \mathrm{P}$, os transtornos mentais causaram mais afastamentos do trabalho que a COVID:

Apesar da pandemia, a covid-ı́ não foi o maior motivo de afastamento de saúde no ambiente de trabalho no último ano. Um levantamento realizado pela $\mathrm{B}_{2} \mathrm{P}$, consultoria especializada no acompanhamento e gestão de funcionários afastados por razões médicas, da It'sSeg Company, mostra que a doença infecciosa ficou em quarto lugar em um ranking que vai de zero a dez. ${ }^{4}$

O levantamento da consultoria inclui um recorte de 331 mil colaboradores em i8 empresas brasileiras. Os dados consideraram os afastamentos entre março de 2020 e março de 202ı. Para o advogado José Carlos Wahle, que defende o aperfeiçoamento dos serviços de medicina ocupacional que acompanham funcionários nos exames admissionais, demissionais e periódicos:

As pessoas estão sobrecarregadas e não se sentem realizadas e compensadas por isso. Além do cansaço, ela tem um sentimento de inutilidade, de que não importa o que se faça, será insuficiente e não leva a lugar nenhum. É um mal contemporâneo, que merece uma justa e correta classificação (...) O exame admissional é um pouco negligenciado hoje, se atendo mais à parte física do que a questões de saúde emocional. As empresas correm o risco de contratar pessoas que estão à beira do Burnout porque tiveram empregos devastadores antes e isso não é detectado no exame admissional. É importante que o exame seja atento e cuidadoso. $^{\text {viii }}$

Segundo Paul Harrison (202I), autor principal do estudo e professor de Psiquiatria da Universidade de Oxford, apesar de o risco dessas doenças ser pequeno do ponto de vista individual, os efeitos na população como um todo são consideráveis para os sistemas de saúde, de assistência social e também para o sistema econômico, pelo fato de que muitas dessas enfermidades são crônicas e o Burnout, sendo uma doença ligada ao trabalho, impacta consideravelmente a performance das empresas. Assim, é crucial o tratamento dessas doenças para o bem-estar da coletividade e para o futuro das companhias públicas e privadas.

\footnotetext{
${ }^{4} \mathrm{O}$ levantamento da consultoria inclui um recorte de $33 \mathrm{I}$ mil colaboradores em $\mathrm{I} 8$ empresas brasileiras e os dados consideraram os afastamentos entre março de 2020 e março de 202I.
} 


\section{IMPACTO DAS DOENÇAS MENTAIS NA COLETIVIDADE}

Não se pode considerar com exatidão uma situação de pós pandemia do Covid-ı́, pois após aproximadamente dois anos seu enfrentamento ainda perdura, podendo ser considerada a maior crise humanitária que essa geração presenciou. De qualquer forma, a cada dia que passa ficam mais evidentes as marcas na população: milhares de mortos, milhares de pessoas afetadas pela dor da perda, por problemas financeiros, pelo afastamento da vida que levavam anteriormente, pelo isolamento social, pelo distanciamento das pessoas, do lazer, do convívio em sociedade.

A saúde mental é uma parte integrante e complementar à manutenção das funções orgânicas do corpo humano e é considerada como um importante fator que possibilita o ajuste necessário para lidar com as emoções positivas e negativas. Nesse contexto, a promoção e a manutenção da saúde mental são essenciais para que o indivíduo tenha a capacidade necessária de executar suas habilidades pessoais e profissionais, sem esse equilíbrio das funções mentais, coloca-se em risco a saúde do convívio social e o exercício dos direitos sociais e de cidadania.

Uma sociedade mentalmente doente está predisposta a sofrer sérios danos prejuízos coletivos. Algumas consequências podem ser apontadas como de cunho geral para toda a população, independente da faixa etária, como, por exemplo, o aumento do número de suicídios. Por outro lado, pode-se atribuir à determinadas faixas etárias ou à determinadas classe de pessoas alguns problemas de ordem específica, por exemplo: o fato de que crianças acometidas de doenças mentais terão, em regra, dificuldade de aprendizado e prejuízos intelectuais, o que impactará no futuro profissional; trabalhadores acometidos de doenças mentais sofrerão impacto da vida profissional e trarão prejuízos tanto à vida pessoal quanto para o empregador; além de diversos outros casos que poderiam ser mencionados como exemplo.

A UNICEF também alertou recentemente acerca da situação mundial da saúde mental de crianças, adolescentes e cuidadores, mencionou os riscos e fatores de proteção em momentos críticos do curso de vida e demonstrou a necessidade de investigar os determinantes sociais que moldam a saúde mental e o bem-estar; afirmou que a pandemia COVID-ıg levantou preocupações sobre a saúde mental de uma geração de crianças, fato 
que pode representar a ponta de um iceberg de saúde mental - um iceberg ignorado por muito tempo. ix

É devastador o resultado previsto como consequência do não tratamento de tais doenças, considerando que elas fazem parte de da vida de mais de 18 milhões de pessoas no Brasil, conforme mostram os dados do quadro abaixo, levantados pela Organização Mundial de Saúde (OMS) no ano de $2017^{\mathrm{x}}$. O país lidera o ranking mundial de transtornos mentais e figura no segundo lugar mundial em casos de depressão: são mais de II milhões de brasileiros com a doença. Não seria o caso de priorizar o problema? De dar a devida importância ao assunto? De estudar, dentro do âmbito de cada entre federado a situação coletiva e implantar medidas realmente eficazes para o enfrentamento e o tratamento desses problemas de saúde mental? Indubitavelmente, a resposta é afirmativa para todas essas indagações.
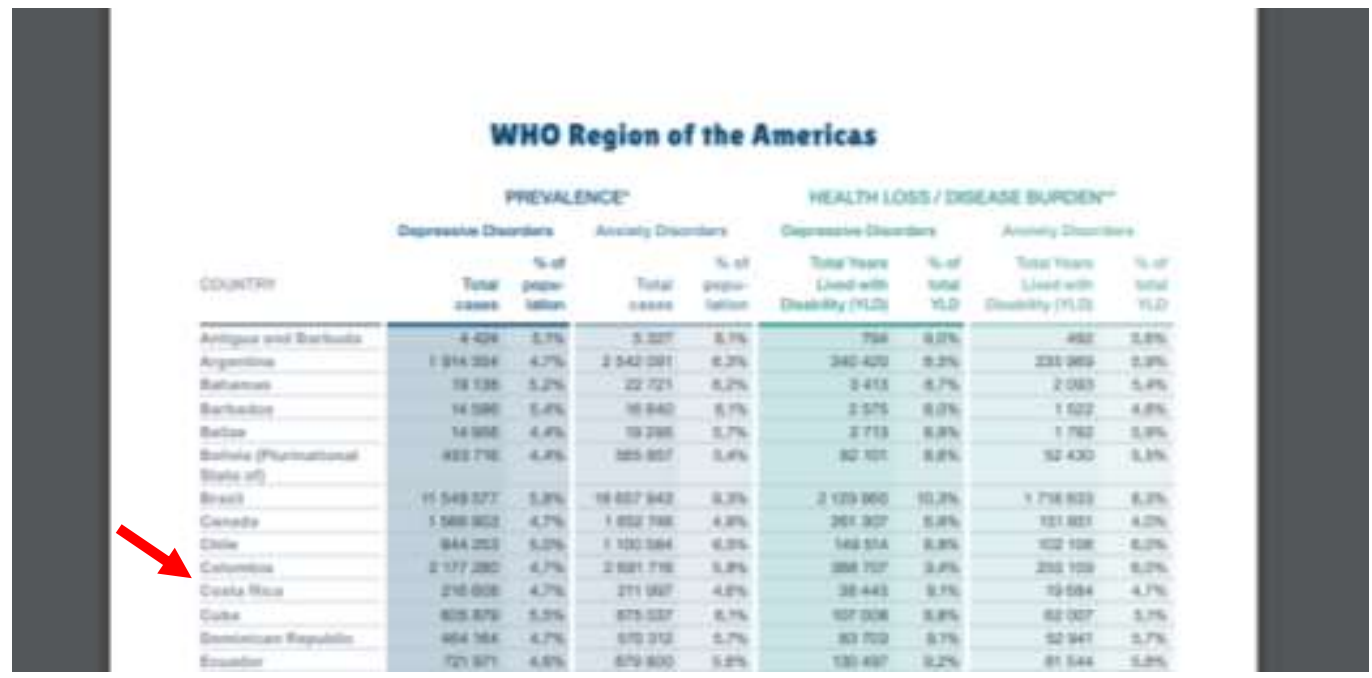

Sendo assim, é inegável a necessidade de atenção ao assunto e consequente criação de políticas capazes de retardar o crescimento de tais doenças, diagnosticar os casos e realizar tratamento adequado. A Administração Pública deve colocar em pauta a necessidade de formular e disponibilizar políticas públicas municipais de tratamento para a retomada do reequilíbrio da saúde mental dos trabalhadores da saúde pública e da população em geral, bem como planejar ações de conscientização da população acerca da importância de buscar tratamento para as doenças e transtornos mentais. 


\section{POLÍTICAS DE SAÚDE MENTAL}

Mesmo antes da pandemia já havia sido identificado um aumento do número de doenças mentais, causadas por diversos fatores, porém, o aumento considerável pode ser atribuído às consequências da pandemia, configurando um dos principais problemas de saúde pública da atualidade e dos anos futuros. As marcas são severas e os profissionais da saúde devem estar preparados para trabalhar com o aumento do sofrimento psíquico que se apresenta há algum tempo e se estenderá no pós-pandemia.

Tal fato preocupa as autoridades, considerando as graves consequências oriundas desse problema de saúde pública. Dentre essas consequências está a redução de contribuições para a economia, já que os transtornos mentais diagnosticados - incluindo Transtorno do déficit de atenção com hiperatividade (TDAH), ansiedade, autismo, transtorno bipolar, transtorno de conduta, depressão, transtornos alimentares, deficiência intelectual e esquizofrenia - podem prejudicar significativamente a saúde, a educação, as conquistas e a capacidade financeira de crianças, adolescentes e jovens no futuro. ${ }^{\mathrm{xi}}$

Sendo assim, a formulação das alternativas para sanar ou equilibrar este problema é uma das mais importantes decisões a ser tomada e implantada pela administração pública, visto que atinge a população de forma generalizada. Para tanto, existem diferentes modelos e formas de tentativa de solucionar os problemas de maneira gradual, buscando resultados eficazes, ainda que a longo prazo, visto a natureza e a complexidade do problema. ${ }^{\text {ii }}$

No Brasil, o atendimento público e gratuito à saúde mental é realizado pelo Sistema Único de Saúde (SUS), sendo direcionado para todo e qualquer cidadão, e pelo Sistema Único de Assistência Social (SUAS), com atividades voltadas às famílias vulneráveis pela pobreza e exclusão social.

Dessa forma, qualquer classe social pode receber as ações em saúde mental nos municípios, entre eles: o atendimento para diagnóstico e acompanhamento contínuo, o acesso à medicação, acolhimento, desenvolvimento de ações de promoção de saúde mental, psicoterapia individual e em grupo, intervenções terapêuticas coletivas, regime de atenção diária, atenção hospitalar e às urgências e reabilitação psicossocial, terapias, além de outros. 
Além do atendimento público, deve haver colaboração do setor privado, através das empresas e do acompanhamento da saúde mental de seus funcionários. Programas que abrangem a conscientização da saúde mental, terapia, consultas médicas, testes psicológicos, meditação, cursos e exercícios diários também devem ser implantados.

Os programas educacionais na área da saúde mental nas empresas devem contar com líderes da saúde para lidar, de forma mais saudável, com as crescentes demandas pessoais, relacionais e organizacionais, considerando os potenciais transtornos psicológicos envolvidos no ambiente de trabalho, com tratamento pautado no aperfeiçoamento das competências socioemocionais, gestão do estresse e das relações sociais, comportamentos tóxicos e formas de lidar com eles, comunicação não violenta e gestão de conflitos no ambiente do trabalho.

O setor público e o setor privado, no intuito de auxiliar nesse resgate do equilíbrio da saúde mental da coletividade, devem trabalhar no sentido de buscar garantir, ao menos minimamente, os meios e os hábitos saudáveis que, conforme o rol do próprio Ministério da Saúde, consistem em: consultar o médico regularmente, tratamento terapêutico, exercícios físicos, atividades intelectuais, reforço nos laços familiares e de amizade, convivência em sociedade e outros.

\section{ऽ IMPLANTAÇÃO DE POLÍTICAS PÚBLICAS}

O Governo Federal possui uma Política Nacional de Saúde Mental, com ação coordenada pelo Ministério da Saúde, que compreende as estratégias e diretrizes adotadas pelo país para organizar a assistência às pessoas com necessidades de tratamento e cuidados específicos em saúde mental, abrangendo transtornos mentais como depressão, ansiedade, esquizofrenia, transtorno afetivo bipolar, transtorno obsessivo-compulsivo e outros. O acolhimento dessas pessoas e seus familiares é uma estratégia de atenção fundamental para a identificação das necessidades assistenciais, alívio do sofrimento e planejamento de intervenções medicamentosas e terapêuticas, se e quando necessárias, conforme cada caso. ${ }^{\text {xiii }}$

O serviço é prestado por meio da Rede de Atenção Psicossocial, formada por várias unidades com finalidades distintas, de forma integral e gratuita, pela rede pública de saúde. 
de problemas relacionados a saúde mental, como exemplo as iniciativas para prevenção do suicídio.

Ainda dentro do Sistema Único de Saúde (SUS), dentre as estratégias de maior relevância para promover o acesso da população ao sistema de saúde, está o Programa de Saúde da Família (PSF), criado em 1994, com o propósito fundamental de fornecer atendimento integral à população. $O$ programa separa o município por territórios e o atendimento é feito por equipes multiprofissionais que acompanham as famílias.

Em conjunto com a equipe de profissionais, vários projetos terapêuticos e interventivos são criados e desenvolvidos, com o objetivo de contribuir no conhecimento e no tratamento da doença mental, com o desenvolvimento de diversas modalidades de trabalhos interdisciplinares, como psicoterapia individual e grupal, intervenções através de grupos interdisciplinares terapêuticos ou de promoção de saúde, como rodas de terapia comunitária, rodas de conversas em saúde mental e outros.

Sendo assim, verifica-se que existe o zelo e a intenção de obter bons resultados com as políticas públicas de saúde mental. Cumpre a cada município, considerando o acesso direto com os cidadãos e considerando os programas previstos pelo SUS, promover a educação coletiva acerca do tema, implantar efetivamente os programas previstos para tratamento da saúde mental, elaborar as leis que se fizerem necessárias para a concretização desse fim, conferindo ao tema a real importância que ele merece.

Para tanto, a administração deve atribuir a devida importância à equipe multidisciplinar que atua na área, incentivando e promovendo a capacitação dos profissionais, focando também nos agentes comunitários, que terão o contato direto com os cidadãos e realizarão a mediação entre as famílias atendidas e os profissionais de saúde.

É notório o fato de que muitos países enfrentam uma escassez de médicos, e no Brasil não é diferente. Além de diversos problemas de estrutura, de escassez de verba e de falta de planejamento, existe um problema de má distribuição geográfica, com demanda por médicos em diversas especialidades. Considerando a tendência no crescimento da utilização do mundo virtual e a significativa evolução da tecnologia da informática, uma alternativa que deve ser estudada pelos entes federativos é a implantação de plataformas 
digitais com recursos criados para minimizar os custos, ampliar o alcance territorial dos atendimentos, otimizar o tempo do profissional e do paciente e auxiliar na continuidade do tratamento. Já existem empresas privadas que usam da tecnologia e da ciência de dados para identificar e rastrear riscos psicológicos nos ambientes corporativos:

Desenvolvemos a primeira plataforma digital de avaliação psicológica do país, a Avax Psi. Hoje, temos ferramentas como o 'Burnômetro', que mede os níveis de exaustão pelo trabalho. Além disso, desenvolvemos o índice de risco psicossocial, que auxilia as empresas a predizer riscos de saúde mental e a implementar planos de ação com base na raiz dos riscos psicossociais e nas necessidades reais dos colaboradores $^{\text {xiv }}$.

Um caso interessante foi implantado no Estado do Mato Grosso do Sul: A Caixa de Assistência dos Advogados do Mato Grosso do Sul (CAAMS) disponibilizou um link de monitoramento digital de saúde mental da advocacia como parte do programa 'LegalMente'.

Inovador, o projeto conta com o apoio da Seccional MS da Ordem dos Advogados do Brasil, Escola Superior de Advocacia (ESA) e visa identificar o perfil da saúde mental dos profissionais da advocacia e diagnosticar as principais demandas e impactos da Covid-19 causados à classe ${ }^{\mathrm{xv}}$.

Tais informações servirão como base para que sejam tomadas as próximas decisões

acerca das ações assistenciais necessárias, orientando o melhor caminho de acordo com as informações coletadas.

Assim, os municípios devem o mais rápido possível iniciar seu planejamento técnico e orçamentário, promover a capacitação dos servidores que atuam na área, editar atos normativos que criem programas e políticas públicas eficazes, dar relevância ao tema e executar sua implantação, acompanhar os resultados e analisar os resultados, promovendo as adaptações necessárias ao longo do tempo.

\section{CONCLUSÃO}

A pandemia da Covid-ı́ não demonstra sinais de estar no fim. Já é tempo de se estudar o que pode ser feito para remediar os danos à saúde mental causados por ela. $\mathrm{O}$ mundo precisa reconhecer o trauma decorrente da pandemia, visto que muitas pessoas estão lutando contra doenças e transtornos mentais resultantes dos traumas deixados por ela. 
O exorbitante número de afastamento de trabalhadores de seus empregos devido aos transtornos mentais reflete em perdas de produtividade para as empresas, prejuízos na qualidade de vida dos trabalhadores, maior índice de acidentes, além de gastos com demandas judiciais, pedidos de demissão e aposentadorias de pessoas em idade altamente produtiva de trabalho. Apesar da existência de algumas normas regulamentadoras do Ministério do Trabalho para a avaliação dos riscos psicossociais, muitas empresas negligenciam essa obrigatoriedade, sendo elas próprias as responsáveis por não prevenir o diagnóstico e a evolução dessas doenças nos seus funcionários.

Aspectos como baixa produtividade e baixo desempenho profissional, escassez de motivação e de ideias, indisposição e faltas no trabalho, ausência de harmonia e cooperação, maiores despesas, aposentadorias precoces, pedidos de demissão, falta de mãode-obra, escassez de pessoas qualificadas e capacitas, dentre outras, são consequências advindas do desequilíbrio da saúde mental. Cuidar desse aspecto da saúde é o primeiro passo para uma vida mais harmoniosa em todos os aspectos, tanto para o trabalhador quanto para a empresa, seja ela privada ou pública.

As alterativas que estão sendo expostas por diversos profissionais devem ser analisadas e implantadas conforme a característica de cada ente, sua possibilidade orçamentária e sua disponibilidade de pessoal, sempre com o devido planejamento e a busca da promoção de uma cultura de saúde mental, de forma sistêmica e duradoura, com ações estratégicas e políticas públicas e organizacionais voltadas a esse fim.

Apesar do aumento na busca de tratamento pelo próprio paciente, é considerável o número de pessoas acometidas por alguma espécie de transtorno mental e que, por diversos motivos e as vezes por falta de conhecimento, não busca ajuda. É aí que o poder público deve agir também, pois a prevenção e o diagnóstico precoce evitarão graves problemas. A criação de políticas públicas de saúde é necessária também para o acolhimento e a facilitação do acesso ao diagnóstico. Para tanto, o engajamento da sociedade, dos órgãos de governo e de especialistas da área médica é fundamental e trará à tona um tema ainda objeto de preconceito e historicamente negligenciado. 


\section{REFERENCIAS}

iSENA, Victor. Transtornos mentais causaram mais afastamentos no trabalho que covid. Disponível em https://exame.com/carreira/transtornos-mentais-afastamentos-trabalhocovid/. Acesso em 19/or/2022.

ii FLORES, J.H. A (re) invenção dos direitos humanos. I $^{\text {a }}$ ed. Florianópolis: Fundação Boiteux, 2009: 26.

iii QUIEROZ, Laísa. Ministério da Saúde - Realidade imposta pela pandemia pode gerar transtornos mentais e agravar quadros existentes, 202I. Disponível em https://www.gov.br/saude/pt-br/assuntos/noticias/202I-I/outubro/realidade-impostapela-pandemia-pode-gerar-transtornos-mentais-e-agravar-quadros-existentes. Acesso em IO/II/202I.

iv TAQUET Maxime, Geddes John R, Husain Masud, Luciano Sierra, Harrison Paul J. 6month neurological and psychiatric outcomes in 236379 survivors of COVID-19: a retrospective cohort study using electronic health records. 2021. Disponível em https://www.thelancet.com/action/showPdf?pii=S2215-0366\%2821\%2900084-5. Acesso em 09/II/202I.

SELIGMANN-Silva, E. Trabalho e desgaste mental: o direito de ser dono de si mesmo. São Paulo: Cortez; 2oIr.

${ }^{v i}$ REEVES A, Mckee M, Stuckler D. Economic suicides in the Great Recession in Europe and North America. Br J Psychiatry. 2014; 205(3):246-247.

vii TAQUET Maxime, Geddes John R, Husain Masud, Luciano Sierra, Harrison Paul J. 6month neurological and psychiatric outcomes in 236379 survivors of COVID-19: a retrospective cohort study using electronic health records. 2021. Disponivel em https://www.thelancet.com/action/showPdf?pii=S2215-0366\%2821\%2900084-5. Acesso em $20 / 01 / 2022$.

viii FUTEMA, Fabiana. Burnout passa a ser classificado como doença ocupacional em 2022. Disponível em https://6minutos.uol.com.br/carreira/burnout-passa-a-ser-classificadocomo-doenca-ocupacional-em-2022-que-muda-na-pratica/?utm_source=thenews \&utm _medium=email\&utm_campaign=referral. Acesso em 23/12/2021.

ix UNICEF, Impacto da covid-ı na saúde mental de crianças, adolescentes e jovens é significativo, mas somente a 'ponta do iceberg' - UNICEF. Disponível em https://www.unicef.org/brazil/comunicados-de-imprensa/impacto-da-covid-I9-na-saudemental-de-criancas-adolescentes-e-jovens. Acesso em I9/II/202I. 
${ }^{x}$ Depression and Other Common Mental Disorders: Global Health Estimates. Geneva: World Health Organization; 2017. Licence: CC BY-NC-AS 3.0 IGO.

${ }^{x i}$ LEAL, Aline. Relatório aponta impacto da pandemia na saúde mental de adolescentes. Disponível em https://agenciabrasil.ebc.com.br/educacao /noticia/202I-1o/relatorioaponta-impacto-da-pandemia-na-saude-mental-de-adolescentes. Acesso em 19/II/202I .

xii RUA, Maria das Graças. Políticas Públicas - Florianópolis: Departamento de Ciências da Administração/UFSC: CAPES: UAB, 2009: 89-9o.

xiii Saúde Mental. https://www.gov.br/saude/pt-br/assuntos/saude-de-a-a-z/s/saudemental. Publicado em 24/II/2020. Atualizado em 30/12/2021. Acesso em 25/or/2022.

xiv TAWIL, Marc. Por que 2022 é o ano da saúde mental nas empresas, e o que a tecnologia tem a ver com isso? Disponível em https://epocanegocios.globo.com /colunas /Futuro-dotrabalho/noticia/2022/ol/por-que-2022-e-o-ano-da-saude-mental-nas-empresas-e-o-quetecnologia-tem-ver-com-isso.html. Publicado em 19/or/2022. Acesso em 26/or/2022.

${ }^{\mathrm{xv}} \mathrm{OAB} / \mathrm{MS}$. 'LegalMente': CAAMS disponibiliza link de monitoramento digital de saúde mental da advocacia. Disponível em http://www.caams.org.br/noticias.php?id=20998 . Publicado em r6/o9/2020. Acesso em 26/or/2022. 